\title{
Stress Relaxation behind Elastic Shock Waves in Rocks
}

\author{
Thomas J. Ahrens \\ Poulter Laboratories, Stanford Research Institute \\ Menlo Park, California \\ George E. Duvall \\ Department of Physics, Washington State University, Pullman
}

\begin{abstract}
The amplitude of elastic shock waves in Arkansas novaculite is observed to decrease at a rate of $\approx 3.3 \mathrm{~kb} / \mathrm{mm}$ for shock propagation path lengths of 6 to $12 \mathrm{~mm}$. The amplitude of the final shock state in the experiments is held near the $155-\mathrm{kb}$ pressure level. A total variation of elastic shock wave amplitude (Hugoniot elastic limit) of $\approx 40 \mathrm{~kb}$ (from 110 to 70 $\mathrm{kb}$ ) is observed in $\approx 1 \mathrm{~cm}$ of shock travel. The intrinsic attenuation term in a constitutive equation for a stress-relaxing elastoplastic material is found to account for $\approx 90 \%$ of the observed peak pressure attenuation of the elastic shock, as compared with the $\approx 10 \%$ which is predicted from the instantaneous elastic shock profile. The Hugoniot elastic limits of Sioux and Eureka quartzites, which were not as intensively studied as the Arkansas novaculite, are also found to decrease with shock propagation path length.
\end{abstract}

\section{INTRODUCTION}

When a large pressure, $p_{\mathbf{g}}$, is applied suddenly to the plane boundary of a rock or other type of solid having a normal pressure-volume curve, the shock wave disturbance that propagates in the $x$ direction forms, in certain stress ranges, two shock wave fronts. The first shock wave front, which has the greater propagation velocity $U_{1}$, is called the elastic shock wave or elastic precursor. This shock is assumed to encompass material which is initially at rest (particle velocity $u=0)$ and at zero pressure ( $p_{x}=$ $p_{y}=0$ ); the shock transforms this material into the elastic shock state. (The $y$ direction is perpendicular to the shock propagation direction.) The elastic shock state has a particle velocity $u=u_{1}$ and longitudinal compressive stress $p_{s}=$ $p_{1}$. The second, or deformational, shock front, propagating at a slower velocity $U_{2}$ with respect to laboratory coordinates, brings the rock from the elastic shock state to the deformational or final shock state. The second shock front imparts a particle velocity $u=u_{2}$ and longitudinal stress $p_{a}=p_{2}$ to the rock. The amplitude of the first shock front is often called the Hugoniot elastic limit and is believed to represent the maximum stress that a rock or other solid material can withstand under conditions of rapid one-dimensional compres- sion without internal rearrangement taking place in the material at the shock front.

As the recording techniques used in determining profiles of shock waves in solids have been refined it has become possible to record a decrease of elastic shock wave amplitude in rocks with increasing propagation path length when the final shock state is at some nearly constant value. The decrease in elastic shock wave amplitude with increasing propagation path length is of special significance for a plane wave system because, in general, when the elastic shock is driven by a second deformational shock in two or three dimensions, the attenuation is due to geometrical spreading. In one dimension, however, the geometrically produced attenuation is absent and the decrease in elastic shock amplitude must reflect a property of the rock rather than be a result of a limitation in the energy available from the shock source. This intrinsic amplitude decrease (or attenuation) of the elastic shock wave has been named elastic wave stress relaxation. In some cases a pronounced increase in elastic shock wave amplitude with increasing final shock state has been observed when the shock propagation path length was fixed. Both an increase in elastic shock wave amplitude with increasing final shock state and a decrease in elastic shock wave 


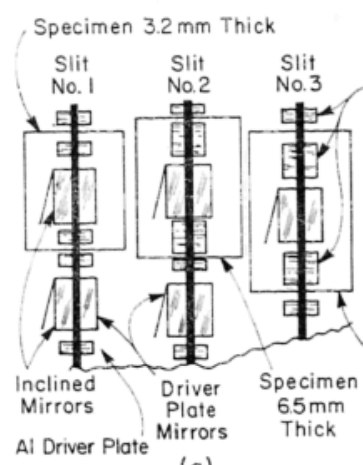

(a)

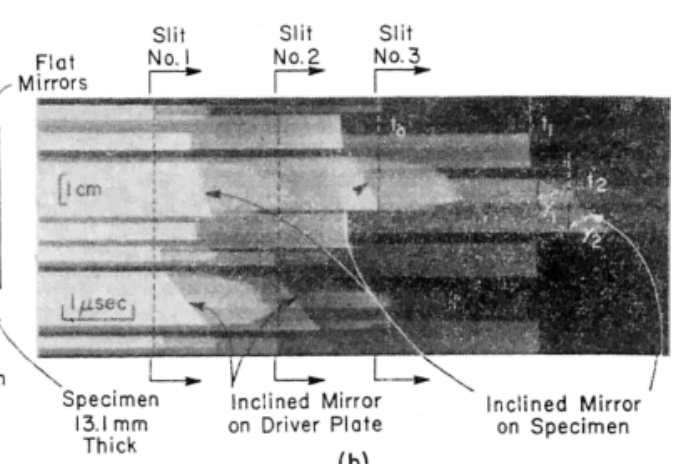

(b)

Fig. 1. Plane wave inclined-mirror streak photograph, shot 10,448. (a) Arkansas novaculite specimen layout. (b) Resulting streak camera record showing elastic and deformational shock wave arrivals. For slit 3 , times indicated by $t_{0}, t_{1}$, and $t_{2}$ correspond to incident shock arriving at driver-plate free surface, elastic shock arriving at specimen free surface, and deformational shock arriving at specimen free surface. Inclined-mirror cutoff angles $\gamma_{1}$ and $\gamma_{2}$ are used to determine the specimen free-surface velocities imparted by elastic and deformational shocks, respectively.

amplitude with increasing shock propagation distance are observed indirectly in Hugoniot determinations in nonporous rocks and minerals [Ahrens and Gregson, 1964]. Strong indications of this phenomenon have been observed in other brittle mediums-single-crystal quartz [Wackerle, 1962; Fowles, 1961], magnesium oxide [Ahrens, 1966], barium titanate [Doran, 1962], and single-crystal germanium [Duvall, 1963]. It has also been observed in body-centeredcubic metals such as Armco iron [Taylor and Rice, 1963].

\section{EXPERIMENTS}

Arkansas novaculite and Sioux and Eureka quartzites were chosen for studying the variation in Hugoniot elastic limit because previous experimentation, some of which was performed before recognition of the stress-relaxation phenomenon, indicated that their elastic limits varied from 50 to $100 \mathrm{~kb}$. Also, the grain size (0.01 $\mathrm{mm}$ in Arkansas novaculite, $0.1 \mathrm{~mm}$ in Sioux quartzite) is small compared with the shock propagation path lengths.

The variation of the elastic shock amplitude can be observed by (1) varying the amplitude of the final shock state or (2) varying the specimen thickness (shock propagation path length) while holding the final shock state constant. We used approach (2) because the experiments are simpler and data can easily be obtained and compared by observing plane wave propagation with both inclined mirror and wedges. The techniques, which are scribed briefly here, are explained in detail b Ahrens and Gregson [1964].

By using either the inclined-mirror or the wedge method we can measure the shock and particle velocities associated with the elasti shock state, $U_{1}$ and $u_{1}$, and those associated rith the deformational shock state, $U_{2}$ and $u_{3 .}$. The Rankine-Hugoniot conservation equations

$$
\begin{gathered}
\rho_{a}\left(U_{b}-u_{a}\right)=\rho_{b}\left(U_{b}-u_{b}\right) \\
p_{b}-p_{a}=\rho_{a}\left(U_{b}-u_{a}\right)\left(u_{b}-u_{a}\right)
\end{gathered}
$$

relate shock stress $p$ (normal to shock troatt density $\rho$, shock velocity $U$, and particle it locity $u$ across the shock front when the $m$ dium is brought from state $a$ to state $b$. Sur. stitution of subscripts 0,1 , and 2 in the equations will refer to the initial zero pressur state, the elastic shock state, and the deform. tional shock state, respectively.

In the inclined-mirror experiments (Figur 1 ), specimens having thicknesses varying fro 3 to $13 \mathrm{~mm}$ were mounted on 19-mm-thick 204 aluminum driver plates. Plane shock wavh nearly flat-topped in pressure profile, were in duced in the driver plates by a plane wart generator initiating a detonation in a combint. tion of high-explosive pads consisting of 13 me of composition B-3 followed by $51 \mathrm{~mm}$ of Bar tol. Flat and inclined mirrors mounted on 


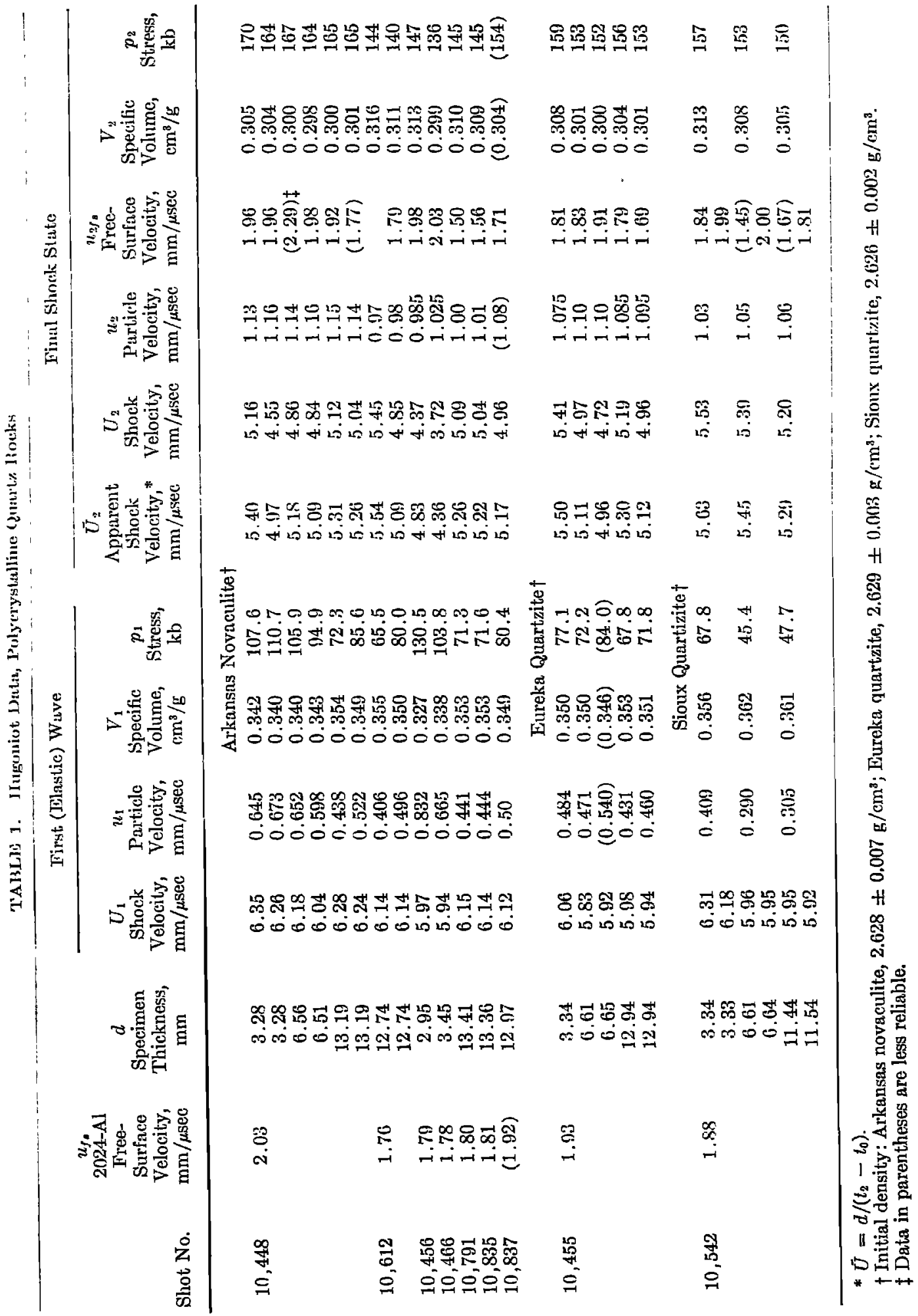




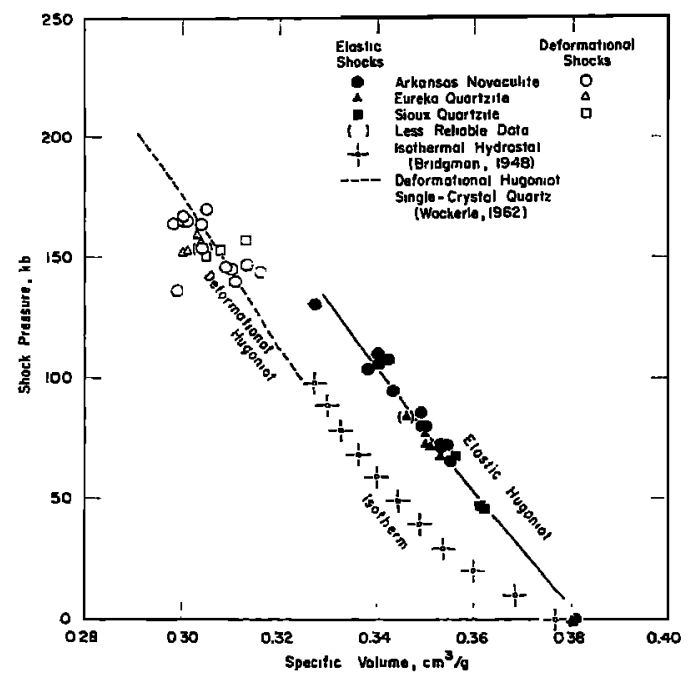

Fig. 2. Hugoniot points for Arkansas novaculite and Sioux and Eureka quartzites obtained in the stress-relaxation experiments.

driver plate and specimen were illuminated by an explosive light source so that the transit times of both shocks through the specimen (thickness, $d$ ) and hence the shock velocities could be measured. The velocities, $u_{h_{f},}$ and $u_{21 s}$, that each of the shocks imparted to the specimen free surface were also measured. The driver plate free-surface velocity was measured with another inclined mirror.

The particle velocity associated with the elastic shock state in the rock is obtained by using the free-surface approximation $u_{1} \cong$ $u_{1 f t} / 2$ [Rice et al., 1958]. Since the shock and particle velocities and the initial density $\rho_{0}$ are known, (1) and (2) are used to determine pressure and density of the elastic shock state. The deformational (second) velocity is calculated from the arrival time $\left(t_{2}\right)$ of a second shock at the free surface, the propagation speed $U_{1}^{\prime}$ of the rarefaction wave produced by the reflection of the elastic shock at the free surface, and the speed of the oncoming deformational shock $U_{:}^{\prime}$ after it has interacted with this rarefaction wave [Fowles, 1961]. spectively. Dispersion of the rarefaction ware (assumed to propagate at $U_{1}^{\prime}$ ) is neglected Essentially identical Hugoniot states were ol culated using several models for material be havior which were thought to be reasonable; each of these gives rise to different formulas for $U_{2}^{\prime}$ and $U_{2}^{\prime}$. The assumption that the disturb. ance which reverberates between the free sur. face and the oncoming second shock travels at a local velocity equal to the initial elastic shock velocity yields the following simple relations:

$$
\begin{aligned}
& U_{1}^{\prime}=U_{1}-u_{1} \\
& U_{2}^{\prime}=U_{1}+u_{1 \rho s}
\end{aligned}
$$

Equations 4 and 5 are used in (3) to calculate $U_{a}$, and from this the deformational shock state is determined by application of the impedane matching method [Rice et al., 1958]. Elasti and deformational Hugoniot states obtained for Arkansas novaculite and Sioux and Eureks quartzites using the plane wave method an given in Table 1 and are plotted in Figure? relative to the Hugoniot [Wackerle, 1962] and isothermal [Bridgman, 1948] data for singte crystals of quartz. In the pressure-volume plane the present data are definitely in agreement; with, although scattered about, the Hugoniot data for single-crystal quartz.

The plane wave wedge technique (Figure 3) was used in three shots. This technique permits determination of the shock and particle velocities, and hence shock pressure, of the elastie shock front as the disturbance propagate through a specimen of variable thickness. In two of these experiments the rate of change of particle velocity behind the elastic shock (before arrival of a deformational shock) was approximately determined. This technique appears more useful in giving the change in the Hugoniot elastic limit with shock propagation path length than in measuring its absolute value.

Different types of light-reflecting metal strips were affixed (Table 2) to the rock specimen

$$
U_{2}=\frac{U_{1}^{\prime}\left[d+u_{1 f_{s}}\left(t_{2}-t_{1}\right)\right]+U_{2}{ }^{\prime} d-U_{1}^{\prime} U_{2}{ }^{\prime}\left(t_{3}-t_{1}\right)}{U_{1}{ }^{\prime}\left(t_{1}-t_{0}\right)+U_{2}^{\prime}\left(t_{2}-t_{0}\right)-u_{1 f_{s}}\left(t_{2}-t_{1}\right)}
$$

where $t_{0}$ and $t_{1}$ are the arrival times of the incident shock at the driver plate-specimen interface and at the upper specimen surface, re- wedges in these experiments. The light image reflected in the metal strips of an explosive grid light source is recorded by a streak camen 


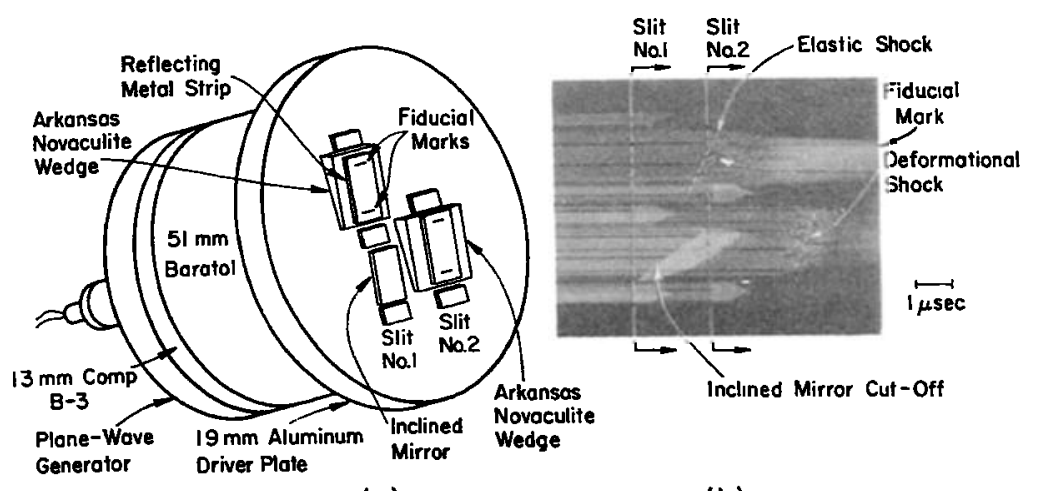

(a)

(b)

Fig. 3. Plane wave wedge experiment. (a) Assembly is viewed along slits 1 and 2 by streak camera. (b) Streak camera record showing grid lines displaced first by elastic shock and then by deformational shock.

(Figure 3). For each type of metal strip the relation of the deflection of the light image, which is a measure of free-surface velocity [Ahrens and Gregson, 1964], and the particle velocity in the underlying rock must be examined at different times. For simplicity the velocity at the free surface of the metal is assumed to be equal to twice the particle velocity of the metal.

The quality of the record for shot 10,464, with a polished aluminum reflecting strip, was the best of the three shots fired. The free-surface relocity measured corresponds to the shock transmitted into the aluminum by incidence of the elastic wave in the novaculite on the noraculite-aluminum interface because the aluminum is thick enough $(3.2 \mathrm{~mm})$ for the rererberation time of a disturbance in the metal to be long ( $\approx 1 \mu \mathrm{sec}$ ) compared with the time in which the wave profile is measured $(0.2$ $\mu$ sec). Specimen particle velocities are obtained graphically (Figure 4).

Since the elastic shock is actually attenuating, the shock pressure inferred from the aluminum will be slightly lower than that in the rock. Because of the close impedance matching between the aluminum and elastic states in Arkansas novaculite, the measured Hugoniot elastic limit and its decrease with shock propagation path length are insensitive to errors arising from the straight-line cross-curve assumption.

The Hugoniot elastic limits shown in Figure 5 and Table 3 all correspond to elastic waves preceding deformational shocks with amplitudes of about $155 \mathrm{~kb}$. The mean decay rate of 3.3 $\mathrm{kb} / \mathrm{mm}$ can be compared with the value 1.4 $\mathrm{kb} / \mathrm{mm}$, at a level of $40 \mathrm{~kb}$ reported for twodimensional geometry by Duvall [1964]. Though the two different measurements cannot be directly compared because of material and geometry differences, it may be considered significant that the results are of the same order of magnitude. The effect of this difference on the relaxation time is to reduce it from the value $2.0 \mu \mathrm{sec}$, reported by Duvall [1964], to approximately $0.7 \mu$ sec.

In shot 10,465 , the particle velocity experimentally obtained for the 0.152 -mm-thick steel strip (shim) is assumed to correspond to the state $F$ (Figure 4) achieved in the steel by transmission of a steady shock at the specimensteel interface. This assumption is made because the initial displacement of the grid image used in calculating the steel free-surface velocity remained nearly steady for $0.04 \mu \mathrm{sec}$ after its initial displacement. This time is of the same order as that $(0.06 \mu \mathrm{sec})$ required for the shock, reflected at the steel free surface, to reflect at the rock-steel interface and again arrive at the free surface. Specular reflectivity of the steel is lost upon the apparent arrival of this (first) reverberation. For this shot the cross curve of the novaculite is again approximated by a straight line of slope $-\rho_{0} U_{1}$ in order to infer the specimen state $S$ from the state $F$ measured for the steel strip. The true form of the cross curve is unknown, and, be- 


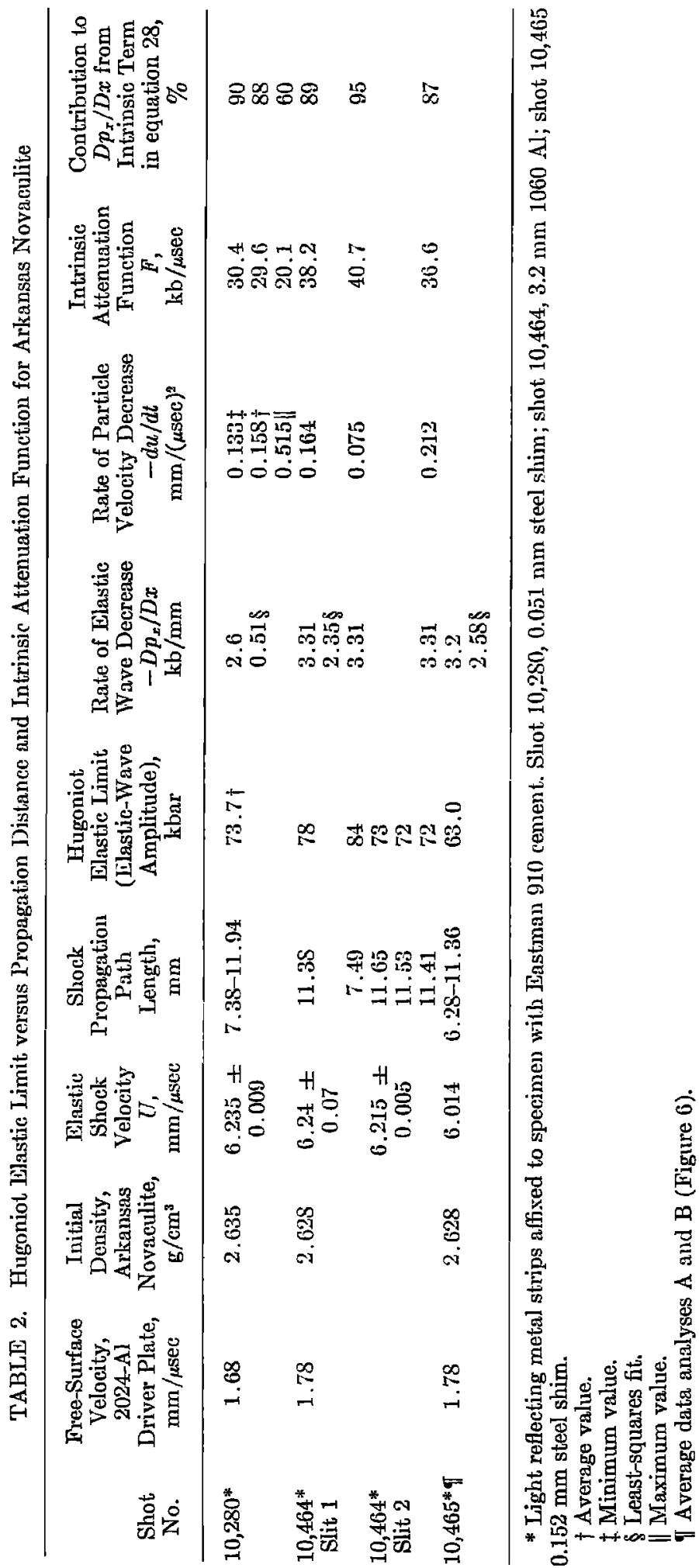




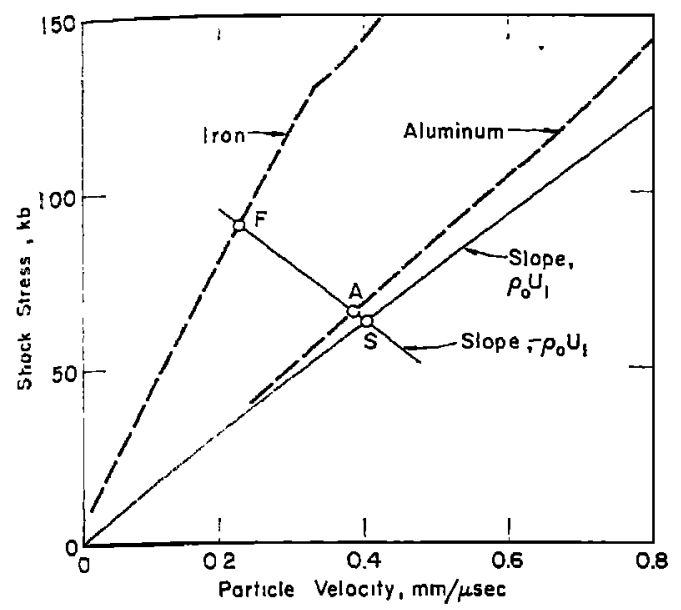

Fig. 4. Relation of shock stress-particle velocity curres of the light-reflecting metal strips to rock elastic Hugoniot state. Intersection of line of slope $-\rho_{0} U_{1}$ drawn through determined states $F$ or $A$, corresponding to steel or aluminum reflecting strips, with line of slope $\rho_{0} U_{1}$ through origin determines (unknown) state $S$ in rock specimen.

cause of the large difference in shock impedances, this approximation may give rise to a significant error in the value obtained for the Hugoniot elastic limit. The two analyses of shot 10,465 (Figure 6) differ because of unusually large uncertainties in the measured angle of grid image displacement $\left( \pm 1^{\circ}\right)$ and camera magnification (nearly $2 \%$ ).

In shot 10,280 , the displacements of the reflected grid images seen in the $0.051-\mathrm{mm}$-thick

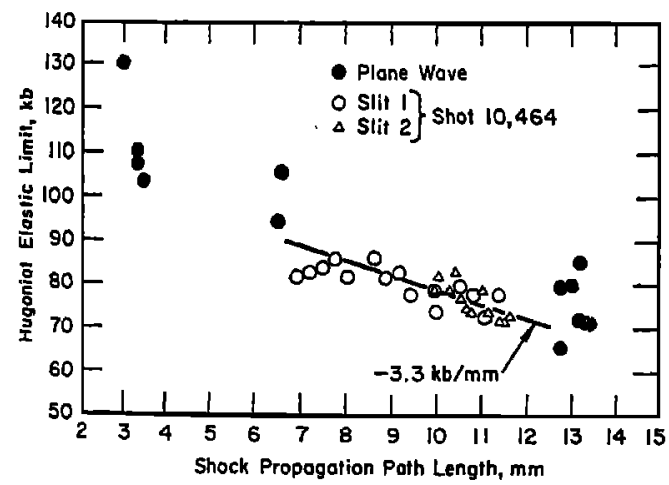

Fig. 5. Hugoniot elastic limit versus shock propagation path length for Arkansas novaculite from shot 10,464 (wedge) and inclined-mirror experiments.
TABLE 3. Hugoniot Elastic Limit versus Propagation Distance for Arkansas Novaculite

\begin{tabular}{|c|c|c|}
\hline Shot No. & $\begin{array}{c}\text { Shock } \\
\text { Propagation } \\
\text { Path Length, } \\
\text { mm }\end{array}$ & $\begin{array}{l}\text { Hugoniot } \\
\text { Elastic Limit } \\
\text { kbar }\end{array}$ \\
\hline 10,464 Slit 1 & $\begin{array}{r}11.38 \\
11.10 \\
10.83 \\
10.55 \\
9.99 \\
9.97 \\
9.44 \\
9.16 \\
8.88 \\
8.60 \\
8.05 \\
7.77 \\
7.49 \\
7.21 \\
6.93\end{array}$ & $\begin{array}{l}78 \\
73 \\
78 \\
80 \\
74 \\
79 \\
78 \\
83 \\
82 \\
86 \\
82 \\
86 \\
84 \\
83 \\
82\end{array}$ \\
\hline 10,464 Slit 2 & $\begin{array}{r}11.65 \\
11.53 \\
11.41 \\
11.17 \\
11.05 \\
10.81 \\
10.69 \\
10.57 \\
10.45 \\
10.33 \\
10.09 \\
9.97\end{array}$ & $\begin{array}{l}73 \\
72 \\
72 \\
74 \\
79 \\
74 \\
75 \\
77 \\
83 \\
79 \\
82 \\
79\end{array}$ \\
\hline 10,465 & $\begin{array}{r}11.36 \\
11.09 \\
10.83 \\
10.60 \\
10.02 \\
9.76 \\
9.49 \\
9.22 \\
8.06 \\
8.69 \\
8.15 \\
7.89 \\
7.62 \\
7.35 \\
7.08 \\
6.55 \\
6.28\end{array}$ & $\begin{array}{l}63.0 \\
69.6 \\
74.2 \\
71.8 \\
72.2 \\
74.3 \\
75.0 \\
73.9 \\
75.9 \\
75.9 \\
82.4 \\
79.0 \\
81.5 \\
78.6 \\
84.9 \\
78.2 \\
76.9\end{array}$ \\
\hline
\end{tabular}

steel shim gave quite scattered values of the steel free-surface velocity (Figure 6 ). After the initial arrival of a shock at the steel free surface, reverberating waves in the steel should, in theory, arrive at the steel free surface at 


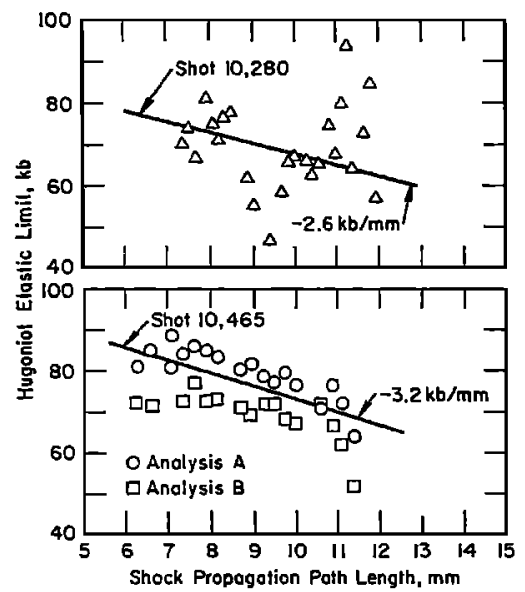

Fig. 6. Hugoniot elastic limit versus shock propagation path length for Arkansas novaculite from shots 10,280 and 10,465 (wedge experiments).

$0.02-\mu \mathrm{sec}$ intervals, until the pressure is reduced to and the particle velocity is increased to that corresponding to a. steady shock in the underlying rock. The differences between the specimen release adiabats which are centered at, for example, states $F$ and $A$ of Figure 4 are neglected. Because of the time resolution of the optical system, these wave arrivals are not separately recorded. A gradual rise in displacement lasting $0.05 \mu \mathrm{sec}$ ( 2 to 3 reverberations) is recorded, however. The image displacements after that time interval are assumed to represent the free-surface velocity that would be achieved by the rock if the reflecting strip were not present. The scatter of these data, as shown

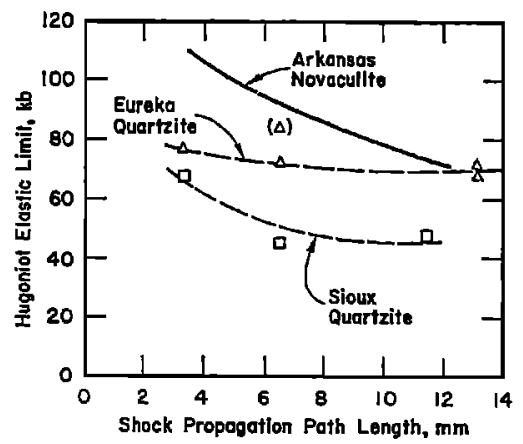

Fig. 7. Hugoniot elastic limit versus shock propagation path length for Eureka and Sioux quartaites. Arkansas novaculite data are shown by solid curve. (Parentheses indicate less reliable datum.)

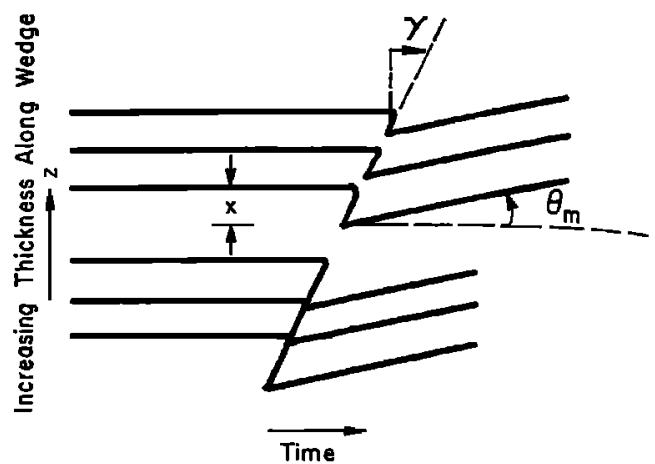

Fig. 8. Diagram of streak camera record of elastic shock arrival for wedge experiment. Angle $\gamma$ and image displacements $x$ determine shock and particle velocity at a series of positions on wedge respectively. Decrease in particle velocity behind elastic shock is calculated by using grid image streak angle $\boldsymbol{\theta}_{\boldsymbol{m}}$.

in Figure 6, is such that no really reliable inference as to decay rate can be made.

Two plane wave inclined-mirror shots with specimens of Sioux and Eureka quartzites were fired to obtain the variation in Hugoniot elastic limit with shock propagation path length (Table 1 and Figure 7). Differences from the Arkansas novaculite curve are suggested by the data but cannot be reliably inferred because of the small number of points and their scattes. If differences do exist, they may be related to differences in grain size-about $0.01 \mathrm{~mm}$ for novaculite and $0.1 \mathrm{~mm}$ for Sioux quartzite.

The rate at which the particle velocity in the rock changes behind the elastic shock front before arrival of the deformational shock cas also be determined by the optical-lever $n$ cording method. In a coordinate system moving with the local particle velocity, the chang of particle velocity, $d u / d t$, is assumed to be proportional to the rate of change of free-surface velocity of the reflecting metal strip affixed to the rock. The rate of change of particle tolocity can then be approximately related to the angle $\theta_{m}$ of the displaced grid image streat (Figure 8) by [Ahrens and Ruderman, 1965]

$$
\frac{d u}{d t} \approx-U_{\mathrm{spD}}\left(\frac{W \theta_{m}}{4 h M_{\mathrm{I}}}+\frac{\partial u_{f s}}{2 \partial z}\right)
$$

(The subscript 1, representing the first shod state, has been dropped.) The angle $\theta_{\mathrm{m}}$ is also related to the rotation of the entire speculats 
reflecting free surface. This rotation is the result of the decrease in free-surface velocity along the wedge face in the direction $z$ of increasing specimen thickness. 1 The angular velocity of the free surface is equal to the gradient of the free-surface velocity $\partial u_{1 s} / \partial z$ (the direction $z$ is approximately perpendicular to the free-surface velocity), $W$ is the streak camera writing rate, $h$ the distance from the grid light source to the reflecting surface, and $M_{1}$ the streak camera magnification of the grid images. $U_{\text {apy }}{ }^{\prime}$ represents the apparent velocity with which rarefaction waves traveling behind the elastic shock intersect the free surface and is given by

$$
U_{\mathrm{app}}{ }^{\prime} \approx U / \sin \alpha
$$

$U_{\text {app }}{ }^{\prime}$ is assumed to be constant for the time interval $(0.2 \mu \mathrm{sec})$ in which the change in particle velocity is to be determined.

The rates of particle velocity decrease given for shot 10,280 in Table 2 represent the lowest, highest, and approximate average of the observed values. For shot 10,464, values of $d u / d t$ were obtained only for the traces that appeared to be least disturbed by fluctuations in the wave profiles. The scatter in these values of $d u / d t$ suggests that extraordinary care and more advanced techniques will be required if more than the sign and magnitude of this parameter are to be obtained experimentally.

\section{THEORY}

A constitutive relation for a stress-relaxing solid of the form

$$
d p_{x} / d t-a^{2}(d \rho / d t)=-F
$$

has been proposed by Duvall [1964]. Here $a$ is the speed of elastic compression waves in material of density $\rho$ and $F$ is a function determining the rate of approach to stress equilibrium, berein called the material's intrinsic attenuation function. In the material model represented by $(\delta), \epsilon_{\sigma}$ and $\epsilon_{m}{ }^{p}$ are total and deformational strain in the $x$ (shock propagation) direction, respectively. The incremental yield strength of this material is defined as

\footnotetext{
${ }^{1}$ This effect was pointed out to us by G. R. Fowles, Stanford Research Institute.
}

$$
d Y=d p_{x}-d p_{y}
$$

For an elastoplastic material, $Y$ will be related to the deformational strain by

$$
d \epsilon_{x}{ }^{p}=\frac{2}{3}\left(d \epsilon_{x}-d Y / 2 \mu\right)
$$

where $\mu$ is the shear modulus. The elastoplastic behavior represented by $(10)$ is one in which the total one-dimensional strain $\epsilon_{x}$ is a sum of an elastic and deformational strain component, the latter representing no net dilatation. Upon noting that $d \epsilon_{x}=d \rho / \rho$ and that the equivalent hydrostatic pressure $\vec{p}=\left(2 p_{y}+p_{x}\right) / 3$, we substitute (9) and (10) into (8):

$$
F=2 \mu\left(d \epsilon_{x}^{D} / d t\right)
$$

$W_{i_{\text {rr }}}$, the irreversible work per unit mass resulting from deformational flow, can be related to the plastic strain by $d W_{i_{x x}}=Y d \epsilon_{x}{ }^{p} / \rho$. Combining this function and (11) shows that $F$ is related to the rate at which irreversible work is performed on the medium:

$$
F=(2 \mu \rho / Y)\left(d W_{\text {irr }} / d t\right)
$$

The theory accounts for attenuation of an elastic shock wave by permitting the material initially to support a higher-than-equilibrium value of shear stress. After the elastic shock front has passed through an element of rock, the shear stress within the element is reduced toward an equilibrium value (which is presumably the static value) by a plastic or fracture process, and forward-traveling rarefaction waves generated within the material overtake the shock front and reduce its amplitude.

In the earlier work of Duvall [1964] a set of attenuation measurements made in two-dimensional geometry were interpreted by a onedimensional theory, and a relaxation time $T$ was calculated, assuming $F=-\Delta p_{s} / T$, where

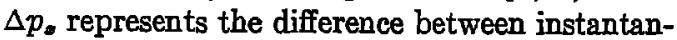
eous and equilibrium stress. Three flaws in this interpretation are: $(a)$ the geometry of experiment and the interpretation did not match; (b) it was necessary to assume a particular form of the function $F$; (c) it was necessary to assume that the equilibrium curve for uniaxial strain is known. In the present interpretation', flaw $(a)$ is corrected and emphasis is placed on calculating a numerical value of $F$ for a particular experimental situation. Assuming (b) and $(c)$, we were able to interpret these results 
in terms of $T$, and this was done for the data plotted in Figure 5; we emphasize, however, that the value of $F$ determined here is independent of assumptions (b) and (c). Recently Taylor [1965] has described stress relaxation in iron by relating $F$ in ( $(\delta)$ to plastic shear strain and dislocation velocity.

The intrinsic attenuation function $F$ in (S) can be evaluated approximately in terms of data obtained in the shock experiments. The time derivatives along both the elastic shock and elastic particle paths are first written, respectively, as

$$
\frac{D}{D t}=U \frac{\partial}{\partial x}+\frac{\partial}{\partial t}
$$

and

$$
\frac{d}{d t}=u \frac{\partial}{\partial x}+\frac{\partial}{\partial t}
$$

Eliminating $\partial / \partial t$ between (13) and (14) and applying the resultant operator to $p_{x}$ and $u$, respectively, yields

$$
\begin{aligned}
D p_{x} / D t & =d p_{x} / d t+(U-u)\left(\partial p_{x} / \partial x\right) \\
D u / D t & =d u / d t+(U-u)(\partial u / \partial x)
\end{aligned}
$$

The mass and momentum conservation equations written in Eulerian coordinates are

$$
\begin{aligned}
d \rho / d t & =-\rho \partial u / \partial x \\
\partial p_{x} / \partial x & =-\rho d u / d t
\end{aligned}
$$

Substitution of $d p_{s} / d t$ from (15) into (S) yields

$$
\frac{D p_{x}}{D t}=a^{2} \frac{d \rho}{d t}+(U-u) \frac{\partial p_{x}}{\partial x}-F
$$

Eliminating $\partial u / \partial x$ between (16) and (17), inserting the result into the first term on the right in (19), and inserting $\partial p_{x} / \partial x$ from (18) into the second term in (19) yields

$$
\begin{array}{r}
\frac{D_{p_{x}}}{D t}=-\frac{a^{2} \rho}{(U-u)}\left(\frac{D u}{D t}-\frac{d u}{d t}\right) \\
-(U-u) \rho \frac{d u}{d t}-F
\end{array}
$$

Eliminating $U$ from (1) and (2) yields, for the case of a single (elastic) shock with the initial state taken at zero pressure,

$$
u^{2}=p_{x}\left(V_{0}-V\right)
$$

Differentiation of (21) along a shock front gives

$$
D u=\frac{V_{0}-V}{2 u}\left[1-\frac{p_{x} /\left(V_{0}-V\right)}{D p_{x} / D V}\right] D p_{x}
$$

Substituting (1) and (2) into (22) and applying the definition of the Hugoniot sound speed

$$
{a^{\prime 2}}^{2}=\left(D p_{s} / D \rho\right)_{\text {Hug }}
$$

and dividing by $D t$ yields

$\frac{D u}{D t}=\frac{V}{2(U-u)}\left[1+\frac{(U-u)^{2}}{a^{\prime 2}}\right] \frac{D p_{x}}{D t}(24)$

Substituting (24) into (20) gives

$$
\begin{aligned}
\frac{D p_{x}}{D t} & =-\frac{a^{2}}{(U-u)}\left\{\frac{1}{2(U-u)}\right. \\
& \left.\cdot\left[1+\frac{(U-u)^{2}}{a^{\prime 2}}\right] \frac{D p_{x}}{D t}\right\} \\
+ & \left\{\frac{a^{2} \rho}{(U-u)}-(U-u) \rho\right\} \frac{d u}{d t}-F
\end{aligned}
$$

Rearranging (25) and using $D x=U D t$ and (2) gives

$$
\frac{D p_{x}}{D x}=\frac{-\rho_{0}\left[(U-u)^{2}-a^{2}\right] \frac{d u}{d t}-\frac{(U-u)^{2}}{U} P}{(U-u)^{2}\left(1+a^{2} / 2 a^{\prime 2}\right)+a^{2} / 2}
$$

Equation 26 expresses the decrense in elastic shock stress with propagation path length in terms of the rate of decrease of mass velocity of the shocked material and the intrinsic attenuation function. A different form of this sesult has been derived by Harris [1950]. With the approximations described below, (26) permits the use of experimental data for evalustion of $F$ and determination of the relative mas nitude of the two terms in the numerator of (26).

To calculate $F$ from experimental values of $d u / d t$ and $D p_{x} / D x$, it is necessary to assume values for $a$ and $a^{\prime}$. One approximation which is convenient is

$$
a \approx a^{\prime} \approx U
$$


This is justified by the relatively small increase 2 density associated with the elastic shock state $(\approx 8 \%)$ and by the close correspondence of the release adiabat and elastic Hugoniot curve [Ahrens and Ruderman, 1965]. Using (27), we ewrite (26) as

$\frac{D p_{x}}{D_{x}} \approx \frac{\rho_{0}\left(2 u U-u^{2}\right) \frac{d u}{d t}-\frac{(U-u)^{2}}{U} F}{3(U-u)^{2} / 2+U^{2} / 2}$

ind solving for $F$ we get

$$
\begin{aligned}
F \approx & \frac{U}{(U-u)^{2}}\left\{\rho_{0}\left(2 u U-u^{2}\right) \frac{d u}{d t}\right. \\
& \left.-\frac{D p_{x}}{D x}\left[3(U-u)^{2} / 2+C^{\prime \prime} / 2\right]\right\}
\end{aligned}
$$

The intrinsic attenuation function $F$ is evaluated by using the values $d u / d t$ and $D p_{x} / D x$ from Table 2 in (29). Fortunately, $F$ depends more on the values of $D p_{x} / D x$ than on the values of $d u / d t$, which at present are poorly determined.

The relative contribution to the observed decrease in elastic shock amplitude with propagation distance can be related to the terms on the right-hand side of (28). The first term gives the contribution to the decrease in shock pressure at the wave front resulting from the change in particle velocity behind the wave front. The second term containing $F$ is the additional contribution to the attenuation of shock stress, which is not accounted for by the particle velocity profile at the preceding instant of time. As is indicated in Table 2, this contribution can account for $90 \%$ of the decrease in peak pressure with propagation distance.

The value of $F$ most representative of the conditions of these experiments is believed to be about $40 \mathrm{~kb} / \mu \mathrm{sec}$. This corresponds to the results shown in Figure 5. The value used for $D_{p_{\mathrm{s}}} / D x,-3.3 \mathrm{~kb} / \mathrm{mm}$, corresponds to that which would be inferred from the results of plane wave inclined-mirror experiments rather than from the least-squares fit to shot 10,464, the latter yielding $-2.35 \mathrm{~kb} / \mathrm{mm}$.

The model proposed by Duvall for an elastopiastic solid involving the function $F$ is probably adequate for deseribing the decay of the elastic precursor in an elastoplastic solid, pro- vided that the proper function is chosen for $F$ and relaxation in the shock front can be neglected. The question whether the elastoplastic formulation can be applied to materials such as rock which may yield by fracture rather than by plastic flow should be studied further.

\section{Summary and Conclusions}

Arkansas novaculite and Sioux and Eureka quartzite specimens shocked to a final (deformational) shock state (near $155 \mathrm{~kb}$ ) display a decrease in elastic shock amplitude (Hugoniot elastic limit) with increasing shock propagation path length. In Arkansas nonvaculite a decrease at the rate of $3.3 \mathrm{~kb} / \mathrm{mm}$ in elastic shock amplitude for shock propagation path lengths of 6 to $12 \mathrm{~mm}$ is observed. A total variation of 40 $\mathrm{kb}$ (from 110 to $70 \mathrm{~kb}$ ) is measured. The Hugoniot elastic limits of Sioux and Eureka quartzites (which are at the somewhat lower stress levels of 50 to $70 \mathrm{~kb}$ and 70 to $80 \mathrm{~kb}$, respectively) were less completely investigated but were also found to decrease with shock propagation path length. Measurements on wedge-shaped specimens in plane wave geometry were used to infer the rate of particle velocity decrease behind the elastic shock front for Arkansas novaculite.

Duvall [1964] has proposed a theory describing the attenuation of elastic shock waves in an elastoplastic material. The observed decrease in elastic shock amplitude with propagation path length is accounted for by assuming that the shocked material will momentarily support a value of shear stress higher than equilibrium. As the shock front passes through an element of rock, the shear stress relaxes toward its equilibrium value and rarefaction waves generated within the material overtake the wave front and reduce its amplitude. The constitutive relation is recast in a form that permits the term corresponding to attenuation to be estimated experimentally from $(a)$ the observed decrease in elastic shock stress with propagation path length and (b) the rate at which a mass element changes its particle velocity immediately after the passage of the elastic shock front (before arrival of the deformational shock front). The intrinsic attenuation term in Duvall's constitutive equation for wave propagation is thus experimentally evaluated. The relative contribution to the decrease in elastic shock 
amplitude from the forward-traveling rarefaction waves overtaking the shock front (corresponding to an observable particle velocity decrease behind the shock front) is compared with the part of the attenuation resulting from the attenuation term in the constitutive relation. It is found that the latter term, corresponding to the intrinsic attenuation, can account for $90 \%$ of the observed decrease of peak pressure of the elastic shock.

Acknowledgments. We appreciate the assistance of M. Ruderman, W. Isbell, and R. White with the experiments. We are also grateful for the scientific discussions and thoughtful criticisms of the manuscript provided by Drs. G. R. Fowles and D. G. Doran.

This research was supported in part through the Defense Atomic Support Agency, contract DA49-146-XZ-277, as part of the Advanced Research Projects Agency's Vela Uniform program.

\section{RETERENCES}

Ahrens, T. J., High pressure electrical behavior and equation of state of magnesium oxide from shock wave measurements, J. Appl. Phys., 57, 2532-2541, 1966.

Ahrens, T. J., and V. G. Gregson, Jr., Shock compression of crustal rocks: Data for quartz, calcite, and plagioclase rocks, J. Geophys. Res., $69,4839-4874,1964$.

Ahrens, T. J., and M. Ruderman, Dynamic properties of rocks, Stanford Res. Inst. Proj. GSU4816, Final Rept. to Defense At. Support Agency, 1965.
Bridgman, P. W., The compression of 39 stan stances to $100,000 \mathrm{~kg} / \mathrm{cm}^{2}$, Proc. Am. Acod. Arts Sci., 76, 55-70, 1948.

Doran, D. G., Basic behavior of ferroelectric in terials under explosively induced shock wate Stanford Res. Inst. Proj. PGD-3484, Final Rept. to Sandia Corp., 1962.

Duvall, G. E., Study of solids at high pressur by dynamic compression, Stanford Res. Init Proj. PGU-2912, Final Rept. to AFOSR, 196\%.

Duvall, G. E., Propagation of plane shock wara in a stress-relaxing medium, in Stress Waves Anelastic Solids, edited by H. Kolsky and Prager, pp. 20-32, Springer-Verlag, Berlin, 1964

Fowles, G. R., Shock wave compression of quarts Ph.D. thesis, Stanford University, June 1961.

Earris, A. J., The decay of plane, cylindrical, and spherical shock waves, in The Shock Wave, $\mathrm{Yol}$ I, Underwater Explosion Research; A cor pendium of British and American Reports, 0 . fice of Naval Research, Washington, D. C., 195

Rice, M. H., R. G. MeQueen, and J. M. Wals Compression of solids by strong shock ware Solid State Phys., 6, 1-63, 1958.

Taylor, J. W., Dislocation dynamics and dynamit yielding, J. Appl. Phys., 36, 3146-3150, 1965.

Taylor, J. W., and M. H. Rice, Elastic-plasix properties of iron, J. Appl. Phys., 34, 364\% 1963.

Wackerle, J., Shock-wave compression of quats J. Appl. Phys., 3s, 922-937, 1962.

(Manuscript received March 7, 1966.) 